\title{
A Case of Primary Aortoduodenal Fistula and Abdominal Aortic Aneurysm in a Patient with Chronic Q Fever
}

\author{
Arne de Niet, MD, Ignace F.J. Tielliu, MD, PhD, Paul M. van Schaik, MD, PhD, \\ Jan J.A.M. van den Dungen, MD, PhD, Clark J. Zeebregts, MD, PhD* \\ Department of Surgery, Division of Vascular Surgery, University Medical Center Groningen, University of Groningen, \\ Groningen, Netherlands
}

\begin{abstract}
A 70-year-old man was successfully treated for an aortoduodenal fistula originating from a $Q$ fever-related abdominal aortic aneurysm. He had no known history of contact with cattle or sheep. Although the combination of abdominal aortic aneurysm and aortoduodenal fistula is rare, one should be suspicious of $Q$ fever infection as the causative agent, and additional medical treatment should be initiated.
\end{abstract}

Copyright $\odot 2017$ Science International Corp.

\section{Key Words:}

Q fever • Aortoduodenal fistula • Aortic aneurysm

\section{Introduction}

A bacterial infection with Coxiella burnetii (C. burnetii) is usually seen in farmers and animal handlers. C. burnetii, also known as $Q$ fever, is transmitted from cattle and sheep to humans by aerosolized particles from urine, feces, or amniotic fluid of contaminated animals [1]. Q fever can present in various manners. Symptoms are mostly mild, with flu-like complaints, headaches, coughing, and nocturnal hyperhidrosis. Sixty percent of infections may be chronic and unrecognized [2]. However, Q fever has also co-occurred with severe diseases, including osteomyelitis, hepatic disease, vascular disease, and

Published by Science International Corp. ISSN 2325-4637

Fax +1 2037853552

E-Mail: aorta@scienceinternational.org

http://aorta.scienceinternational.org

endocarditis [2-4]. In 1988, the first case of an abdominal aortic aneurysm (AAA) associated with polymerase chain reaction (PCR)-proven $\mathrm{Q}$ fever was described [5]. Here, we present a case of a patient with a primary aortoduodenal fistula and AAA concomitant with chronic $Q$ fever infection.

\section{Case Presentation}

A 70-year-old male presented to the emergency department with collapse and dizziness. He had a medical history of gout, percutaneous transluminal angioplasty of the right superficial femoral artery due to claudication, appendectomy, and inguinal hernia operation. There was no history of duodenal ulcers, known AAA, or any known contact with cattle or sheep. After collapse, the patient had melena without abdominal pain, nausea, or dyspnea. Physical examination showed a pulse of 70 beats per minute, blood pressure of $135 / 75 \mathrm{mmHg}$, respiratory rate of 16 breaths per minute, and a temperature of $36.2^{\circ} \mathrm{C}$. $\mathrm{His}$ abdomen was not tender or suspicious. Palpable pulses were present at the level of the common femoral arteries. Melena was seen upon rectal examination. The patient's serum hemoglobin decreased while in the emergency department from 9.8 to $6.9 \mathrm{mmol} / \mathrm{L}$. Colonoscopy did not reveal a bleeding focus. Computed tomography angiography (CTA)

* Corresponding Author:

Clark J. Zeebregts, MD, PhD

Department of Surgery Division of Vascular Surgery,

University Medical Center Groningen

P.O. Box 30.0019700 RB Groningen, The Netherlands

Tel.: +31 503613382; Fax: +31 503611745; E-Mail: c.j.a.m.zeebregts@umcg.nl 
showed an AAA of $54 \mathrm{~mm}$ with the duodenum in close proximity but without a blush of contrast into the duodenal lumen (Figure 1, Figure 2, Figure 3). Nevertheless, an aortoduodenal fistula was suspected, and an operation was scheduled. A median laparotomy was performed, which showed a fistula between the aorta and duodenum (Figure 4, Figure 5).

Samples of the aortic wall were taken and sent for pathology examination. The duodenal fistula was primarily closed with double sutures. The AAA was replaced by a rifampicin-soaked Dacron aorta-biiliac

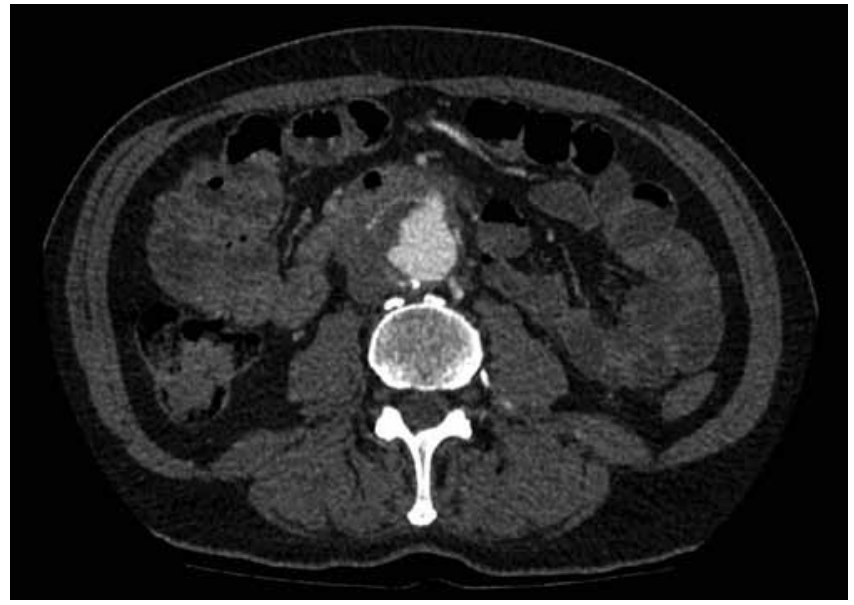

Figure 1. Axial computed tomography angiography image showing an abdominal aortic aneurysm of $54 \mathrm{~mm}$ with a fistula to the duodenum.

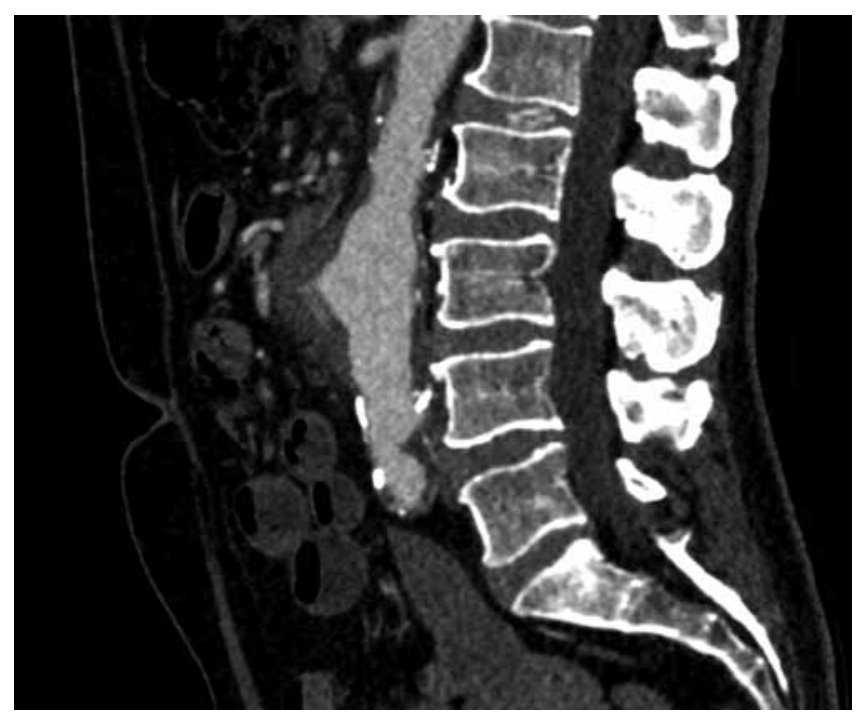

Figure 2. Sagittal computed tomography angiography image showing a fistula on the anterior side of the aorta.

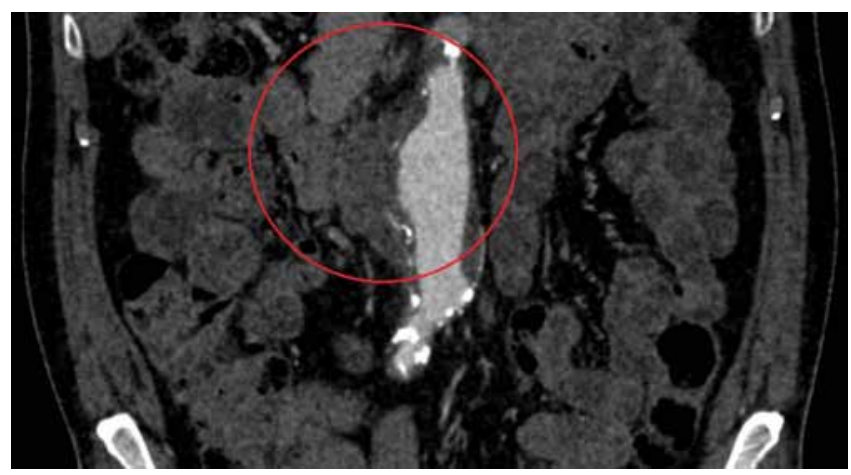

Figure 3. Coronal CTA image showing the aorta, the AAA of $54 \mathrm{~mm}$, and its relation to the duodenum (within the red circle).

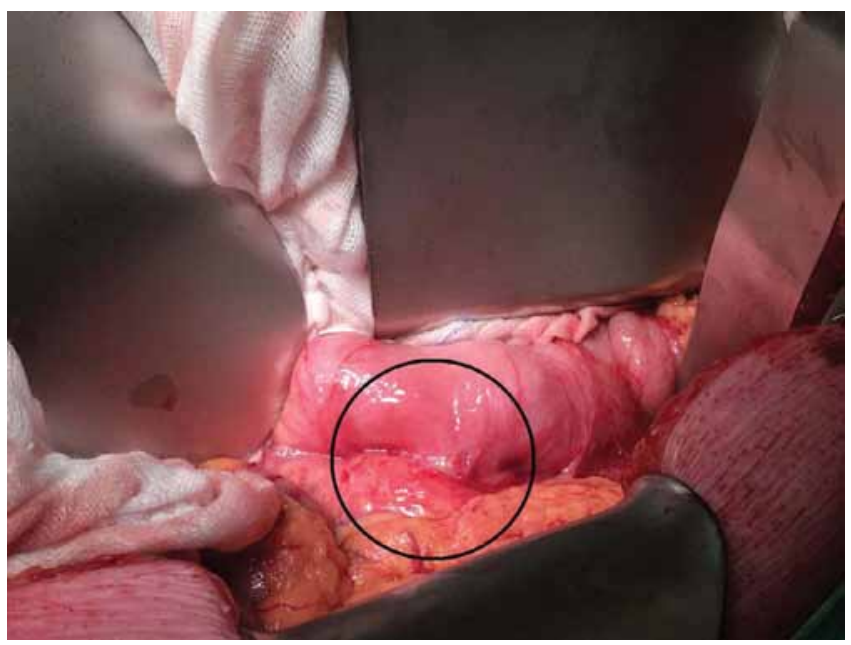

Figure 4. Intraoperative image of suspected aortoduodenal fistula seen when opening the retroperitoneum.

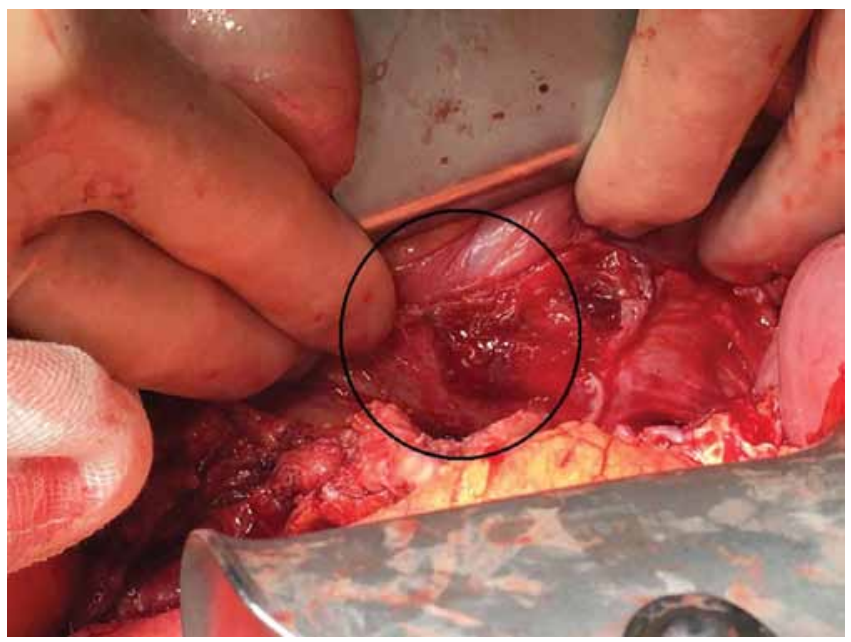

Figure 5. Intraoperative image of an aortoduodenal fistula seen from inside the aorta when opened. 
prosthesis, which was covered by an omentoplasty. During the operation, an enteral feeding tube was placed distal to the duodenal ulcer. Pathology examination of the aortic wall showed a slight inflammatory response and fibrosis. In tissue cultures, no bacteria were found, but positive IgG anti-C. burnetii phase 1 and 2 antiglobulin serology was found. IgM anti- $C$. burnetii phase 2 antiglobulin was negative, suggesting a chronic $Q$ fever infection. Seven days after the first set of positive antiglobulins, serology was repeated, which confirmed the diagnosis of chronic $Q$ fever infection. Antimicrobial therapy with $100 \mathrm{mg}$ doxycycline and $200 \mathrm{mg}$ hydroxychloroquine twice a day was started. Endocarditis was excluded by transesophageal echocardiogram. The patient was dismissed from the hospital 15 days after admission and was doing well at the last outpatient clinic visit 2 months later.

\section{Discussion}

Our case describes the successful treatment of a patient with $\mathrm{Q}$ fever-related vascular disease. Although the combination of AAA and aortoduodenal fistula is rare, one should be suspicious of $Q$ fever infection as the causative agent, and additional medical treatment should be initiated.

\section{Conflict of Interest}

The authors have no conflict of interest relevant to this publication.

\section{Comment on this Article or Ask a Question}

\section{References}

1. Mejia A, Toursarkissian B, Hagino RT, Myers JG, Sykes MT. Primary aortoduodenal fistula and $\mathrm{Q}$ fever: an underrecognized association? Ann Vasc Surg. 2000;14:271-273. DOI: 10.1007/ s100169910046

2. Schneeberger PM, Wintenberger $C$, van der Hoek W, Stahl JP. Q fever in the Netherlands - 2007-2010: what we learned from the largest outbreak ever. Med Mal Infect. 2014;44:339-353. DOI: 10.1016/j.medmal. 2014.02.006

3. Leahey PA, Tahan SR, Kasper EM, Albrecht M. Chronic Q-Fever (Coxiella burnetii) causing abdominal aortic aneurysm and lumbar osteomyelitis: a case report. Open Forum Infect Dis. 2015;3:ofv185. DOI: 10.1093/ofid/ofv185

4. Sessa C, Vokrri L, Porcu P, Maurin M, Stahl $\mathrm{JP}$, Magne JL. Abdominal aortic aneurysm and Coxiella burnetii infection: report of three cases and review of the literature. J Vasc Surg. 2005;42:153-158. DOI: 10.1016/j. jvs.2005.03.022

5. Micoud M, Brion JP, Boulard JC, Magne JL, Gratacap B, Stahl JP, et al. Infection of aortic aneurysm with Coxiella burnetii. Lancet. 1999;338:584. DOI: 10.1016/j. jvs.2005.03.022
Cite this article as: de Niet $A$, Tielliu IFJ, van Schaik PM, van den Dungen JJAM, Zeebregts CJ. A Case of Primary Aortoduodenal Fistula and Abdominal Aortic Aneurysm in a Patient with Chronic Q Fever. AORTA (Stamford). 2017;5(1):27-29. DOI: http://dx.doi. org/10.12945/j.aorta.2017.16.043 